\title{
Structural characterization of boron-doped submicron vapor-grown carbon fibers and their anode performance
}

\author{
K. Nishimura, Y.A. Kim, T. Matushita, T. Hayashi, and M. Endo ${ }^{\text {a) }}$ \\ Faculty of Engineering, Shinshu University, 500 Wakasato, Nagano 380-8553, Japan \\ M.S. Dresselhaus \\ Massachusetts Institute of Technology, Cambridge, Massachusetts 02139
}

(Received 29 September 1999; accepted 23 March 2000)

\begin{abstract}
Structural evolution of undoped and boron-doped submicron vapor-grown carbon fibers (S-VGCFs) was monitored as a function of heat-treatment temperature (HTT). Based on x-ray and Raman data, over the range of HTT from 1800 to $2600{ }^{\circ} \mathrm{C}$, it was found that boron atoms act as catalysts to promote graphitization due to boron's higher diffusivity. For the range of HTT from 2600 to $2800{ }^{\circ} \mathrm{C}$, the process of boron out-diffusion from the host material induces defects, such as tilt boundaries; this process would be related with the improved capacity and Coulombic efficiency of boron-doped S-VGCFs. When $10 \mathrm{wt} \% \mathrm{~S}$-VGCFs was used as an additive to synthetic graphite, the cyclic efficiency of the capacities was improved to almost $100 \%$.
\end{abstract}

\section{INTRODUCTION}

Vapor-grown carbon fibers (VGCFs) have been grown by the decomposition of hydrocarbons, such as benzene and methane, using transition metal particles as a catalyst at a growth temperature of 1000 to approximately $1300{ }^{\circ} \mathrm{C} .{ }^{1-7}$ These fibers have been characterized in terms of the highly preferred orientation of their graphitic basal planes parallel to the fiber axis, with an annular ring texture in the cross section. This structure gives rise to excellent mechanical properties, very high electrical and thermal conductivity, and a high graphitizability of the fibers. ${ }^{4,5}$ Therefore, many researchers have tried in recent years to reach the final target of mass production. Among the various processing methods, the most important one has been the development of the floating reactant method, ${ }^{6-8}$ which allows a three-dimensional dispersion of the hydrocarbon together with the catalytic particles derived from the pyrolysis of organometallic compounds, such as ferrocene, in a reaction chamber, resulting in a high yield and a rather uniform diameter of the resulting fibers. Therefore, the floating reactant method is thought to be a promising means for the mass production of carbon fibers at relatively low cost. The fibers obtained by the floating reactant method in our laboratory have a narrow diameter distribution (from 0.1 to $0.2 \mu \mathrm{m}$ ), showing almost the same morphology as compared to that of normal vapor-grown carbon fibers (VGCFs) (10 to approximately $20 \mu \mathrm{m}$ ), which consist of a central filament and an external deposit of pyrolytic carbon with the an-

\footnotetext{
a) Address all correspondence to this author. e-mail: endo@endomoribu.shinshu-u.ac.jp
}

nular ring structure of a tree. ${ }^{4}$ Furthermore, these fibers have a high possibility for application as a filler in composites and as an anode material in lithium ion batteries, including additives to anode materials, due to their excellent conductivity and high surface-to-volume ratio.

Since boron doping into carbon materials has been known to change the physical and chemical properties, there have been many in-depth studies of the synthesis method, oxidation behavior, and atomic and electronic structure of boron-doped carbons. ${ }^{9-14}$ Generally, it is agreed that a small number of boron atoms can enter the graphite lattice through a substitution reaction, possibly changing the microstructure of the original carbon material. ${ }^{15,16}$ In particular, it has been reported that the use of boron-doped carbon materials as an anode material in lithium ion batteries shows good cyclic efficiency and a little higher capacity than that of undoped carbon materials. ${ }^{17}$

Therefore, in this study, the microstructural deviations of the fibers caused by boron doping, relative to that of the undoped fibers, as a function of heat-treatment temperature (HTT) was monitored by various techniques, such as x-ray diffraction, field-emission scanning electron microscopy (FE-SEM), and microprobe Raman spectroscopy. Since the boron-doping process takes place starting from the near-surface of carbon materials, the Raman technique provides a sensitive tool for monitoring the boron-doping process, especially focusing on the boron-induced disorder in the host material.

Finally, the anode performance and powder conductivity of undoped and boron-doped submicron vaporgrown carbon fibers (S-VGCFs) were evaluated, and when used as additives in synthetic graphite, the cyclic 
efficiency was measured as a function of the weight percent (wt\%) of S-VGCFs added to the graphite host material.

\section{EXPERIMENTAL}

The fibers used in this study were grown by the decomposition of benzene using ferrocene as the catalyst, and the detailed synthesis conditions are shown elsewhere. ${ }^{18}$ Boron doping for the fibers was performed using boron carbide as the dopant, and the boron carbide had an average diameter of $26 \mu \mathrm{m}$ (Wako Pure Chemical Industries, Ltd., Osaka, Japan). Three wt $\%$ of boron carbide and fibers were mixed for $20 \mathrm{~s}$ using an electric mixer, and this mixture was carbonized and graphitized at specific HTT for $10 \mathrm{~min}$ in a high-purity argon atmosphere $(99.999 \%)$.

X-ray diffraction (XRD), polarized Raman scattering, and FE-SEM characterization of the samples was carried out as a function of HTT. An x-ray diffractometer (Rigaku RINT 2100, 40 kV, 30 mA; Tokyo, Japan) with a $\mathrm{Cu} \mathrm{K}_{\alpha}(\lambda=1.54056 \AA)$ source was used to obtain the Bragg reflections from the fibers. The fiberlike powder was pressed on an off-axis-oriented quartz plate using petroleum jelly as the binder. Also, the petroleum jelly was used as a standard for calibrating the scattering angles, because the standard shows sharp paraffin (110) and (200) diffraction lines at $21.482^{\circ}$ and $23.849^{\circ}$, respectively. ${ }^{19}$ The half-width half-maximum (HWHM) linewidth and interlayer spacing $\mathrm{d}_{(002)}$ of the fibers were determined from the width and positions of the (002) diffraction lines, respectively.

Raman scattering spectra were taken at room temperature under ambient conditions using a Reinshaw Raman Image Microscope System 1000 (Gloucestershire, U.K.), equipped with a charge-coupled device (CCD) multichannel detector. The excitation source was a 514.5-nm Ar-ion laser line. The laser beam was focused to about an $0.8-\mu \mathrm{m}$ spot on the fiber surface using a microscope objective of $\times 100$. The optical power at the sample was maintained at $10 \mathrm{~mW}$ to avoid peak shifting of the Raman spectra and to prevent fiber damage due to local overheating. In particular, we obtained Raman spectra with two different geometries; one with the incident light polarized perpendicular to the scattering plane and the scattered light analyzed in the same direction (hereafter denoted as the parallel geometry with intensity $I_{\|}$), the other with the incident light polarized perpendicular to the scattering plane and the scattered light analyzed parallel to the scattering plane (hereafter denoted as the vertical geometry with intensity $I_{\perp}$ ). The peak frequencies of the Raman spectra were determined by fitting the raw data with Lorentzian distribution functions. Thus, the peak positions of the Raman lines were obtained with an accuracy better than $\pm 1 \mathrm{~cm}^{-1}$. Raman spectra obtained with the two different scattering geometries, respectively, yielded the depolarization ratio $\left(D_{\mathrm{p}}=\mathrm{I}_{\perp} / I_{\|}\right)$for the $\mathrm{E}_{2 \mathrm{~g} 2}$ mode, which is expressed as $D_{\mathrm{p}}=1$ for an ideal crystal and $3 / 4$ for polycrystalline material. ${ }^{20}$ The measured values of $D_{\mathrm{p}}$ were calibrated against reference peaks of carbon tetrachloride, which show complete depolarization $\left(D_{\mathrm{p}}=0.75\right)$ at the 218 - and $314-\mathrm{cm}^{-1}$ Ramanactive lines of $\mathrm{CCl}_{4}{ }^{21}$

The powder resistivity and the performance of the undoped and boron-doped S-VGCFs as an anode material in litherium ion batteries were evaluated. Finally, when used as an additive to synthetic graphite, the variation of the cyclic efficiency of synthetic graphite was measured as a function of the added weight percent of $\mathrm{S}-\mathrm{VGCFs}$. Electrochemical measurements were performed using three-electrode test cells made of Pyrex glass. The working electrode was prepared by mixing graphitized S-VGCFs or a mixture of graphitized S-VGCFs and synthetic graphite at $95 \mathrm{wt} \%$ with poly (tetrafluoroethylene) (PTFE) of $5 \mathrm{wt} \%$ as a binder. The pastelike mixture of about $1 \mathrm{mg}$ was spread thinly onto a nickel mesh $(5 \times 5 \mathrm{~mm})$ and pressed at a pressure of 200 $\mathrm{kg} / \mathrm{cm}^{2}$. The electrolyte used was a $1-\mathrm{M}$ solution of $\mathrm{LiPF}_{6}$ in a mixture of ethylene (EC) and diethyl carbonate (DEC). The specific capacity of the electrode was measured in the range 0 to $1.5 \mathrm{~V}$, with a current density of $0.2 \mathrm{~mA} / \mathrm{cm}^{2}$ using a potentiostat/galvanostat (Hokuto Denko Co., Ltd., HA-15) during the charge and discharge cycles.

\section{RESULTS AND DISCUSSION}

The fibers used in this study have a relatively uniform diameter from 0.12 to $0.2 \mu \mathrm{m}$ and a smooth surface, as shown in the FE-SEM photography of Fig. 1(a). The ratio of the fiber length to diameter (the aspect ratio) is on the order of several tens. A very interesting feature of this fiber is its tip morphology, which has a little larger diameter as compared with that of the uniform fibers, and sometimes consists of two, three, or more particles, like the petals of a flower. The tip structure, including the carbide compound found at the tip, have been extensively investigated because its composition and morphology bear a strong relation to the growth mechanism of VGCFs. ${ }^{3,4}$ It is understood that the composition of the carbide compound at the tip changes as a function of temperature. That is, based on the phase diagram of $\mathrm{Fe}-\mathrm{FeC}_{x}$ (which describes the solubility of carbon in $\mathrm{Fe}$ ), the small size of the carbide compound at the reacting site would become larger with decreasing temperature due to the decreased solubility of carbon in Fe with cooling after the reaction. From the above feature of the tip morphology in relation to the growth conditions, it is expected that the most important factor necessary to get a good morphology for the resulting VGCFs is how to 


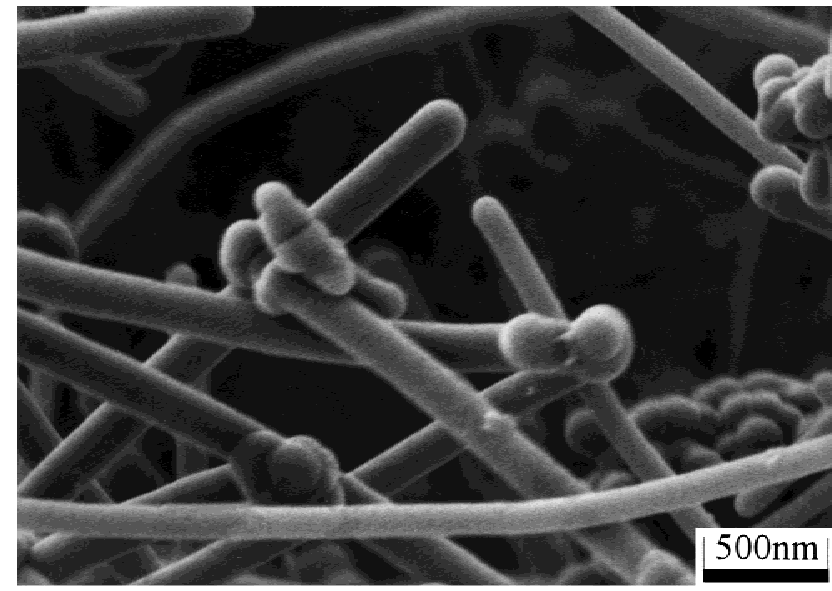

(a)

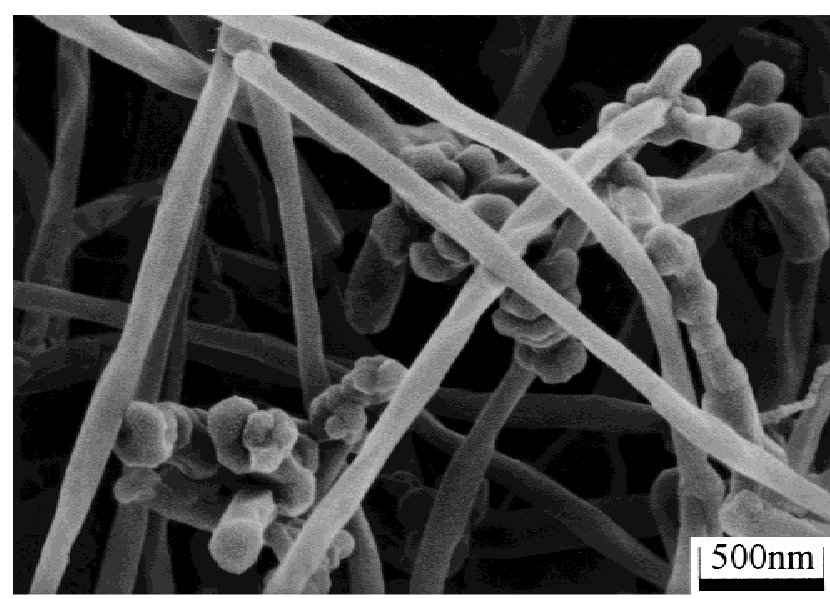

(b)

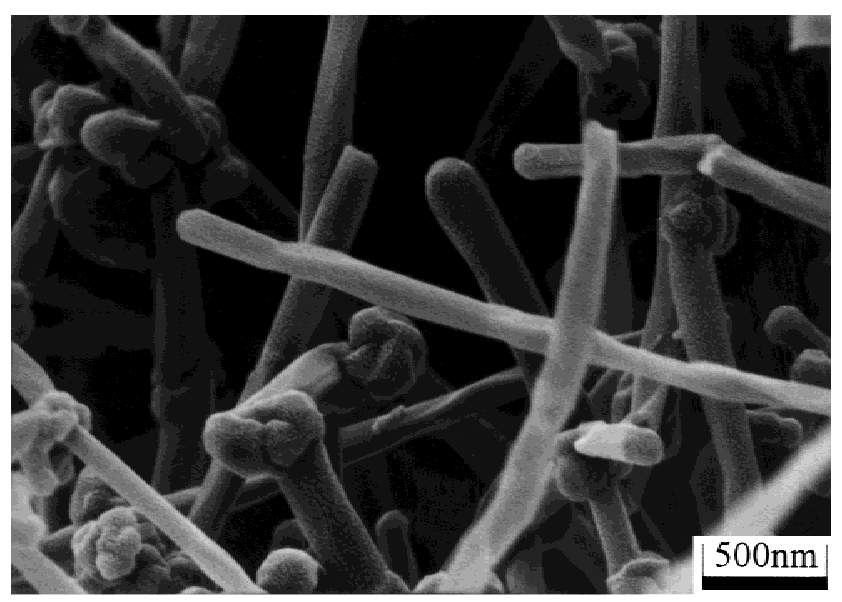

(c)

FIG. 1. FE-SEM photographs of (a) the as-prepared S-VGCFs, (b) S-VGCFs at HTT $=3000{ }^{\circ} \mathrm{C}$, and (c) boron-doped S-VGCFs at $\mathrm{HTT}=3000^{\circ} \mathrm{C}$.

produce a good dispersion of the catalyst with the optimum concentration of the organometallic compound used under these experimental conditions.

No difference in microstructure between the borondoped samples and the undoped samples at $3000{ }^{\circ} \mathrm{C}$ was found (see Fig. 1), maybe due to the low resolution of FE-SEM photography. The interesting feature about Fig. 1 is the faceting phenomena of the surface and tip parts of the fibers, which were systematically studied previously using dark-field transmission electron microscopy. ${ }^{4}$ The results were explained by the flattening of the layer plane, which is initiated at the edge of the fiber; then, the flattening of the layers propagates toward the center of the fibers, and the schematic microstructural evolution of the fiber cross section was suggested by the progressive HTT. As a result, the driving force for this phenomenon is the release of strain energy in the transformation from a curved to a flat layer.

Figures 2(a) and 2(b) shows x-ray diffraction (XRD) patterns for undoped and boron-doped S-VGCFs, respectively. The $2 \theta / \theta$ angle range from $20^{\circ}$ to $28^{\circ}$ contains the graphite (002) diffraction peak and includes the narrow (110) and (200) diffraction lines from the paraffin standard at $21.482^{\circ}$ and $23.849^{\circ}$. For the case of the undoped fibers, the diffraction line at around $26^{\circ}$ is upshifted as HTT increases, and the intensity of this line also increases with increasing HTT, indicative of typical graphitizable carbon materials. On the other hand, the dispersion of the diffraction line for boron-doped fibers as a function of HTT is very different. Transformation from the very broad (002) diffraction line of the borondoped fibers for HTT $=1800{ }^{\circ} \mathrm{C}$ into a very sharp diffraction line for HTT $=2000{ }^{\circ} \mathrm{C}$ indicates that the boron atoms act as a graphitization catalyst, as previously reported. ${ }^{15-17}$ The intensity of the (002) diffraction line increases with increasing HTT and reaches a maximum for the sample with HTT $=2600{ }^{\circ} \mathrm{C}$, after which the intensity gradually decreases with increasing HTT.

To monitor the deviation of the structural evolution of $\mathrm{S}-\mathrm{VGCFs}$ due to boron doping relative to the undoped S-VGCFs, the structural parameters, such as the interlayer spacing and the HWHM linewidth, for undoped and boron-doped S-VGCFs, respectively, are shown in Fig. 3. The interlayer spacing $\mathrm{d}_{\mathrm{OO2}}$ of undoped S-VGCFs decreases gradually with increasing HTT, and finally approaches $3.375 \AA$ for the sample at $\mathrm{HTT}=3000{ }^{\circ} \mathrm{C}$, and the value of $\mathrm{d}_{\mathrm{OO2}}$ is a little higher than that of normal VGCFs $(3.35 \AA)$ with a diameter of $\sim 20 \mu \mathrm{m} .{ }^{3}$ The most plausible explanation for this phenomenon is in terms of a size effect caused by the increased strain energy, as previously reported. ${ }^{4}$ It is also expected that the starting temperature of the faceting phenomena in the S-VGCFs would be lower than that of normal VGCFs, and this is confirmed by the variation of the Raman spectroscopic parameters discussed below. On the other hand, the boron-doped S-VGCFs show a drastically lower value of the interlayer spacing and HWHM of the $\mathrm{d}_{\mathrm{OO2}}$ diffraction linewidth, as compared with that of S-VGCFs. The abrupt decrease of the interlayer spacing from HTT = 1800 to $2000{ }^{\circ} \mathrm{C}$ indicates that the boron diffusion proc- 

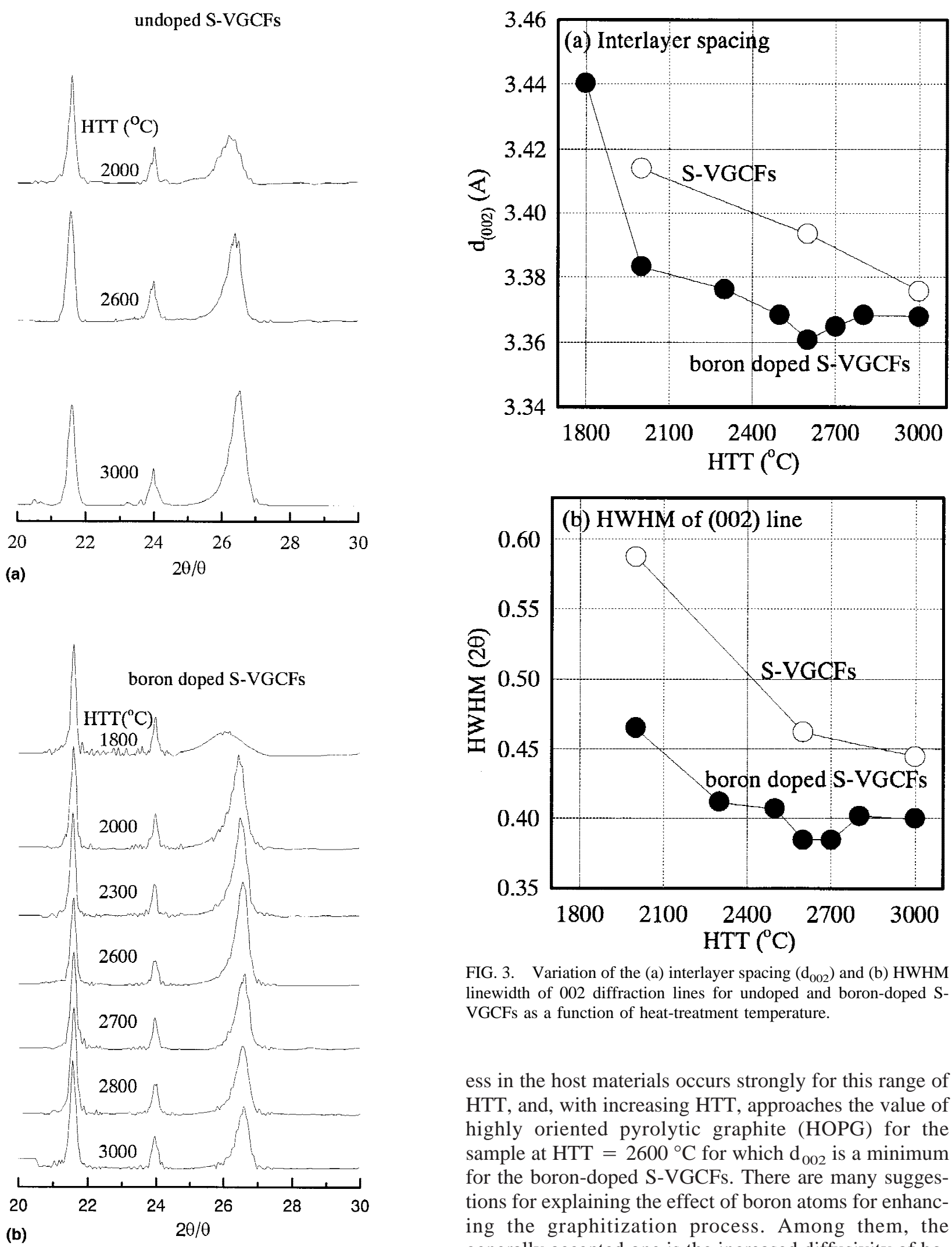

FIG. 3. Variation of the (a) interlayer spacing $\left(\mathrm{d}_{002}\right)$ and (b) HWHM linewidth of 002 diffraction lines for undoped and boron-doped $S$ VGCFs as a function of heat-treatment temperature.

ess in the host materials occurs strongly for this range of HTT, and, with increasing HTT, approaches the value of highly oriented pyrolytic graphite (HOPG) for the sample at $\mathrm{HTT}=2600^{\circ} \mathrm{C}$ for which $\mathrm{d}_{002}$ is a minimum for the boron-doped S-VGCFs. There are many suggestions for explaining the effect of boron atoms for enhancing the graphitization process. Among them, the generally accepted one is the increased diffusivity of boron relative to carbon atoms in the lattice, and this dif-

FIG. 2. XRD patterns of (a) undoped S-VGCFs and (b) boron-doped fusivity is 76 times higher for boron than for carbon atoms for the $a$ direction and 4 times higher for the $c$ 
direction. $^{22}$ The interesting feature of these data is the tendency of the interlayer spacing and its associated HWHM linewidth to increase for the range of HTT from 2600 to $2800{ }^{\circ} \mathrm{C}$, and this increase in $\mathrm{d}_{002}$ and HWHM can be explained by the process of conversion of boron atoms from substitutional to interstitial site for some fraction of the boron atoms in the lattice and also the out-diffusion of boron atoms from the host materials to some extent. Both processes would induce a disruption to the graphene crystalline ordering, accompanying the formation of an increasing number of lattice defects. Finally, the ordering in the graphene sheet would be expected to recover with the further increasing of HTT, and some portion of the defects would then be annealed out due to the increased mobility of the carbon atoms, as is seen for the sample with HTT $=3000{ }^{\circ} \mathrm{C}$. As a result, boron atoms act as a graphitization catalyst for the HTT range from 1800 to $2500{ }^{\circ} \mathrm{C}$ due to their higher diffusivity relative to carbon atoms, followed by the decreasing solubility of boron in the host materials as HTT further increases. Thus, with increasing HTT, the substitutional boron atoms would become unstable due to their decreased solubility, so that the boron atoms would diffuse out of the lattice to induce an increasing number of lattice defects in the host material.

Raman microprobe spectroscopy has been extensively used to characterize various carbon materials, especially showing a high sensitivity for disorder. ${ }^{23,24}$ In this study, polarized Raman scattering spectra were obtained using different geometries for the parallel and vertical directions, respectively. Figures 4(a) and 4(b) show polarized Raman scattering spectra for undoped and boron-doped S-VGCFs, respectively. Single-crystal graphite only shows the Raman-allowed $\mathrm{E}_{2 \mathrm{~g} 2}$ mode at $1580 \mathrm{~cm}^{-1}$. Most imperfect carbon materials show additional peaks at around $1350 \mathrm{~cm}^{-1}$ and in some cases also near $1620 \mathrm{~cm}^{-1}$. These kinds of peaks are due to a defect-induced mode, which can be interpreted in terms of a relaxation of the wave-vector selection rule due to finite crystallite sizes within the spot size of the laser Raman beam. ${ }^{25}$ As seen in Fig. 4, three peaks are observed for all samples. The Raman spectra for the undoped S-VGCFs show almost a similar behavior as compared with that of normal VGCFs with a diameter of $\sim 20 \mu \mathrm{m} .{ }^{23}$ On the other hand, the boron-doped S-VGCFs show almost the same spectra independent of HTT over the entire HTT range from 1800 to $3000{ }^{\circ} \mathrm{C}$ including upshifted $\mathrm{G}$ and $\mathrm{D}$ peaks and the distinct appearance of $\mathrm{D}^{\prime}$ peaks at around $1620 \mathrm{~cm}^{-1}$. For a more detailed analysis of the Raman spectra, curve fitting of the spectra was performed using Lorentzian distribution functions to fit each of the features in the spectra.

Figures 5(a) and 5(b) show the variation with HTT of the relative intensity $\left(I_{\mathrm{D}} / I_{\mathrm{G}}\right)$ for undoped and borondoped samples, respectively. For the HTT range from
1800 to $3000{ }^{\circ} \mathrm{C}$, the relative intensities for the borondoped samples varied only a little in the range from 0.75 to 0.5 for both polarizations parallel and vertical, as shown in Fig. 5(b). On the other hand, the undoped samples show a gradual decrease in $I_{\mathrm{D}} / I_{\mathrm{G}}$ with increasing HTT, especially between 2000 to $2600{ }^{\circ} \mathrm{C}$. Since the relative intensity $\left(I_{\mathrm{D}} / I_{\mathrm{G}}\right)$ is strongly related to the crystallite size, it is seen that structural disorder is induced by the boron-doping process. In terms of the variation of the crystallite size with HTT, it is better to refer to the depolarized ratio $\left(I_{\mathrm{V}} / I_{\mathrm{P}}\right)$ for both samples (Fig. 6). The depolarized ratio of the $\mathrm{G}$ peak for HOPG is unity whereas that of polycrystalline graphite is about $3 / 4 .^{20}$ The undoped samples show a nearly linear increase in $D_{\mathrm{p}}$ as a function of HTT, indicating a monotonic increase in crystallite sizes with increasing HTT. On the other hand, the boron-doped samples show the same tendency for the samples with HTT below $2300{ }^{\circ} \mathrm{C}$; and then they start to deviate from the undoped S-VGCF behavior for HTT near $2500{ }^{\circ} \mathrm{C}$; then they approach a minimum value in $D_{\mathrm{p}}$ for the sample with HTT $=2700{ }^{\circ} \mathrm{C}$; and finally $D_{\mathrm{p}}$ increases once again with increasing HTT. These kinds of behavior can be explained by the doping process of boron into the host material, the process of boron outdiffusion, and the subsequent structural restoration with increasing HTT. As a result, the substitutional boron atoms become unstable due to the decreased solubility of boron, and thus the boron atoms diffuse out from the host material. This kinetic process creates defects such as point defects due to vacancies that cause a decrease in the $D_{\mathrm{p}}$ of the $\mathrm{G}$ peaks for the sample at HTT $=2700{ }^{\circ} \mathrm{C}$. Finally, the disrupted structure caused by the boron outdiffusion process is eventually restored through an annealing process due to the increased mobility of the carbon atoms in boron-doped S-VGCFs. These kinds of physical behavior are consistent with the results of the XRD measurements (Fig. 3). Even though the optical skin depth of the laser excitation for the Raman spectra and the detection depth of the x-ray are very different, the similar results reported here are related to the very small diameter $(\sim 0.2 \mu \mathrm{m})$ of the S-VGCFs.

The variation of the G-band peak frequencies and the frequency difference between the $G$ and $D$ peaks are shown in Figs. 7(a) and 7(b), respectively. As you can see, the $G$ peaks of the undoped samples show a monotonic decrease with increasing HTT, and finally coming close to the value of the G-peak frequency in singlecrystal graphite $\left(\sim 1582 \mathrm{~cm}^{-1}\right)$. On the other hand, the $\mathrm{G}$ peaks of the boron-doped samples show at first a small increase in peak frequency for the range below $1800{ }^{\circ} \mathrm{C}$; followed by a decrease up to $\mathrm{HTT} \approx 2500{ }^{\circ} \mathrm{C}$; and then an abrupt increase for the sample with HTT $=2600{ }^{\circ} \mathrm{C}$; and finally a further decrease in the G-peak frequency with increasing HTT. The frequency difference between the G and $\mathrm{D}$ peaks for the boron-doped samples show a rela- 

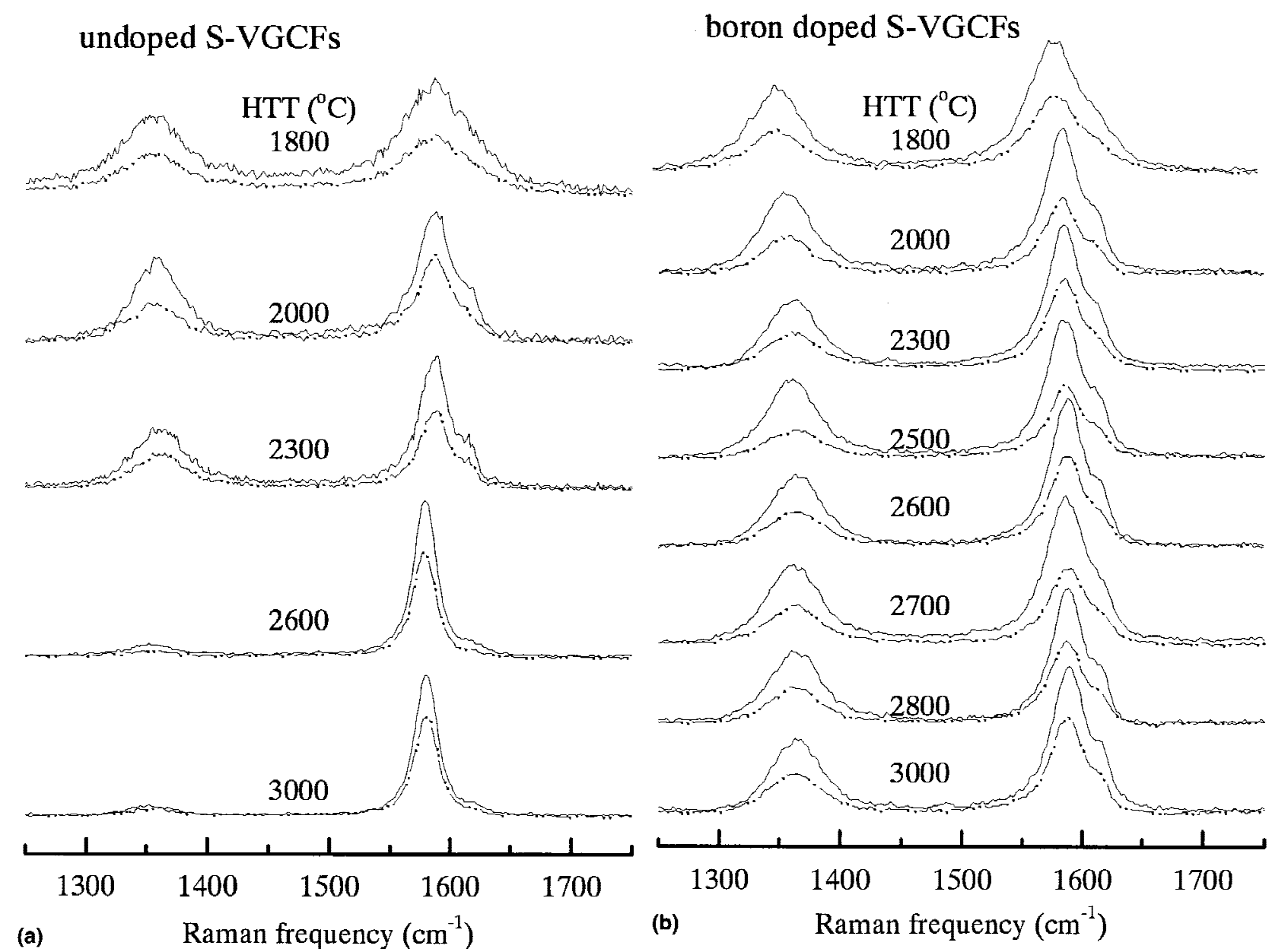

FIG. 4. Polarized first-order Raman scattering of (a) undoped S-VGCFs and (b) boron-doped S-VGCFs for the HTT range from 1800 to $3000{ }^{\circ} \mathrm{C}$. Solid curves indicate the parallel polarization geometry, whereas the dotted curves indicate the vertical polarization geometry for the polarized Raman laser.

tively lower value as compared with that of the undoped samples. This displacement of the curves in Fig. 7(b) can be explained by a deviation in the phonon dispersion relations caused by the substitutional boron atoms in the graphene layers. As a result, the upshifted $G$ peaks for the boron-doped samples would be related to the deviation of the phonon dispersion curves, while the abrupt upshift of the $\mathrm{G}$ peak for the sample at HTT $=2600{ }^{\circ} \mathrm{C}$ might be related to the disorder caused by the boron out-diffusion process.

It is well known that the HWHM linewidths of the G and $\mathrm{D}$ peaks are related to the disorder of the carbon materials. As shown in Fig. 8, the boron-doped samples generally have a higher value for the HWHM linewidths as compared with those of the undoped samples. This indicates that boron atoms act as a defect creator. In particular, the HWHM linewidth of the G peak for the sample with $\mathrm{HTT}=2700{ }^{\circ} \mathrm{C}$ shows a maximum value, consistent with the out-diffusion of boron from the host material which occurs at this HTT.
When the boron carbide was used as a dopant, byproducts were formed, as shown in Fig. 9(a). A small particle is shown for the sample at HTT $=2300{ }^{\circ} \mathrm{C}$; this particle would aggregate more boron carbide with increasing HTT to form larger particles [Fig. 9(b)]; and, finally, these aggregated particles become faceted [Fig. 9(c)]. The formation of this byproduct is related to the recrystallization of boron carbide, which might be different compared with that of the original boron carbide particles used to supply boron for doping. As a result of the outdiffusion of the substitutional boron atoms at about $2500{ }^{\circ} \mathrm{C}$, boron carbide particles can form because the manufacturing temperature of boron carbide is known to be $2500{ }^{\circ} \mathrm{C}$.

Figure 10 shows the variation of the powder resistivity for both the undoped and boron-doped samples with HTT $=3000{ }^{\circ} \mathrm{C}$ as a function of powder density. Here, the boron-doped samples show a relatively lower resistivity, possibly due to two effects: (i) the boron atoms being substitutional in the graphene layer act as an elec- 

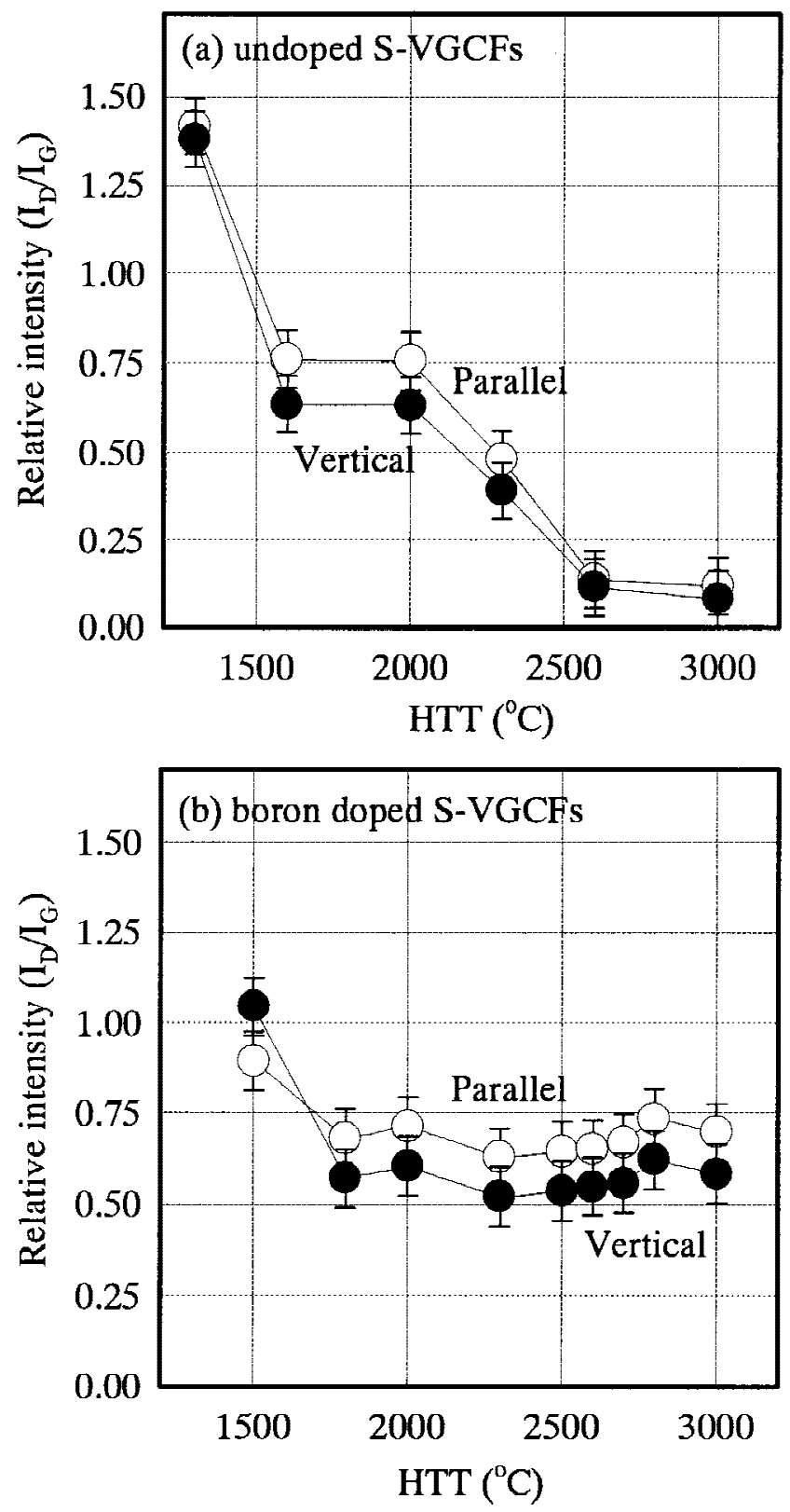

FIG. 5. The variations of the relative intensities of the $\mathrm{D}$ peak to the $\mathrm{G}$ peak $\left(I_{\mathrm{D}} / I_{\mathrm{G}}\right)$ for (a) undoped S-VGCFs and (b) boron-doped SVGCFs as a function of heat-treatment temperature for the parallel and vertical polarization geometries.

tron acceptor, and (ii) the presence of an increased fraction of defects. Figure 11 shows charge and discharge curves for (a) undoped and (b) boron-doped S-VGCFs samples at HTT $=3000{ }^{\circ} \mathrm{C}$ at the first cycle. As compared with that of the undoped sample, the boron-doped samples show a relatively higher charge-discharge capacity, and also better Coulombic efficiency. It is possible to explain the increased capacity caused by boron doping in terms of the boron atoms in substitutional sites and by the increase in the density of defects, such as tilt boundary defects, which contribute strongly to the XRD

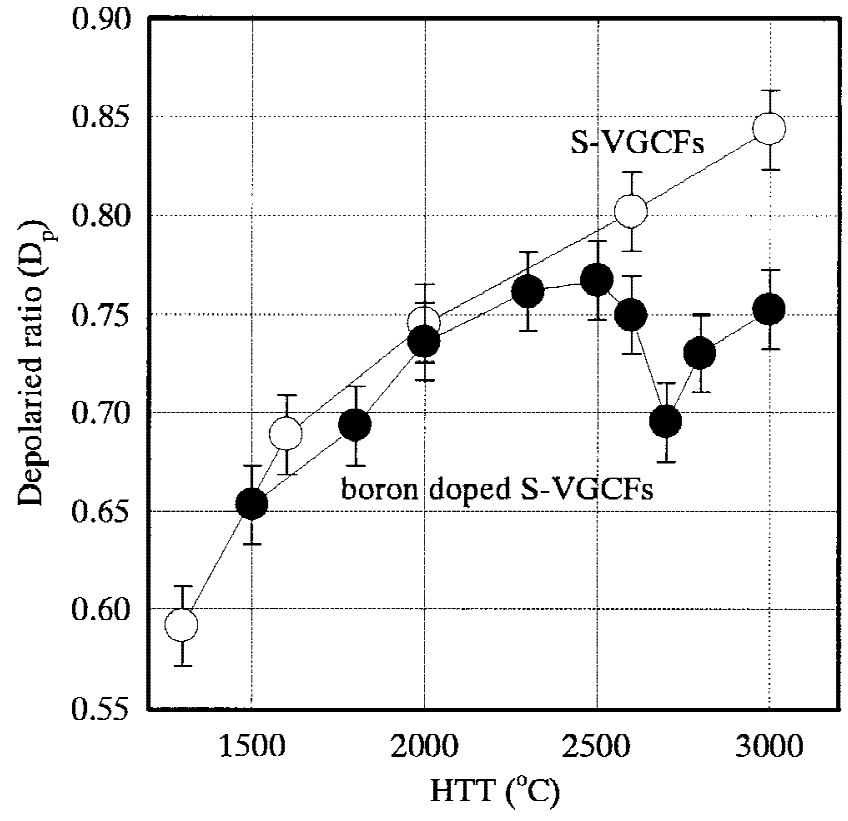

FIG. 6. Variation of the depolarized ratio $\left(D_{\mathrm{p}}=I_{\mathrm{V}} / I_{\mathrm{P}}\right)$ of the $\mathrm{G}$ peaks for undoped and boron-doped S-VGCFs as a function of heattreatment temperature (see text).

results. Up to now, the cyclic efficiency through boron doping is known to increase, whereas the capacity of boron-doped samples depends on the characteristic of the host materials. For the case of the S-VGCFs, the borondoping process induces a higher capacity due to the particular characteristics of these fibers.

The S-VGCFs used in this study are typical discontinuous carbon fibers with a high conductivity, and thus have high potential for use as an additive to an anode material in a lithium ion battery. When used as an additive, the distribution of fiber length is a very important factor for determining the mixing property between the additive and the anode material. Figure 12 shows the results for the length distribution of an undoped S-VGCF sample heat-treated at $3000{ }^{\circ} \mathrm{C}$ obtained by a laser particle size analyzer. The results of Fig. 12 show a wide length distribution for the S-VGCFs, which can be divided into two regions in the criteria of $\sim 2 \mu \mathrm{m}$. Though the mixing effect of S-VGCFs depends also on the type of carbon anode materials that are used, it is expected that the fiber corresponding to the region $\geqslant 2 \mu \mathrm{m}$ would effectively act as an additive to interconnect the anode carbon particles. Figure 13 shows the cyclic efficiency of synthetic graphite $\left(\mathrm{HTT}=2900{ }^{\circ} \mathrm{C}\right)$ as a function of added weight percent of S-VGCFs (HTT $=3000{ }^{\circ} \mathrm{C}$ ). With increasing added weight percent of S-VGCFs, the cyclic efficiencies of synthetic graphite were increased continuously, and in particular when $10 \mathrm{wt} \%$ of SVGCFs was added, the cyclic efficiency was maintained at almost $100 \%$ for many cycles. In terms of the effects of the S-VGCFs added, increased conductivity and elec- 

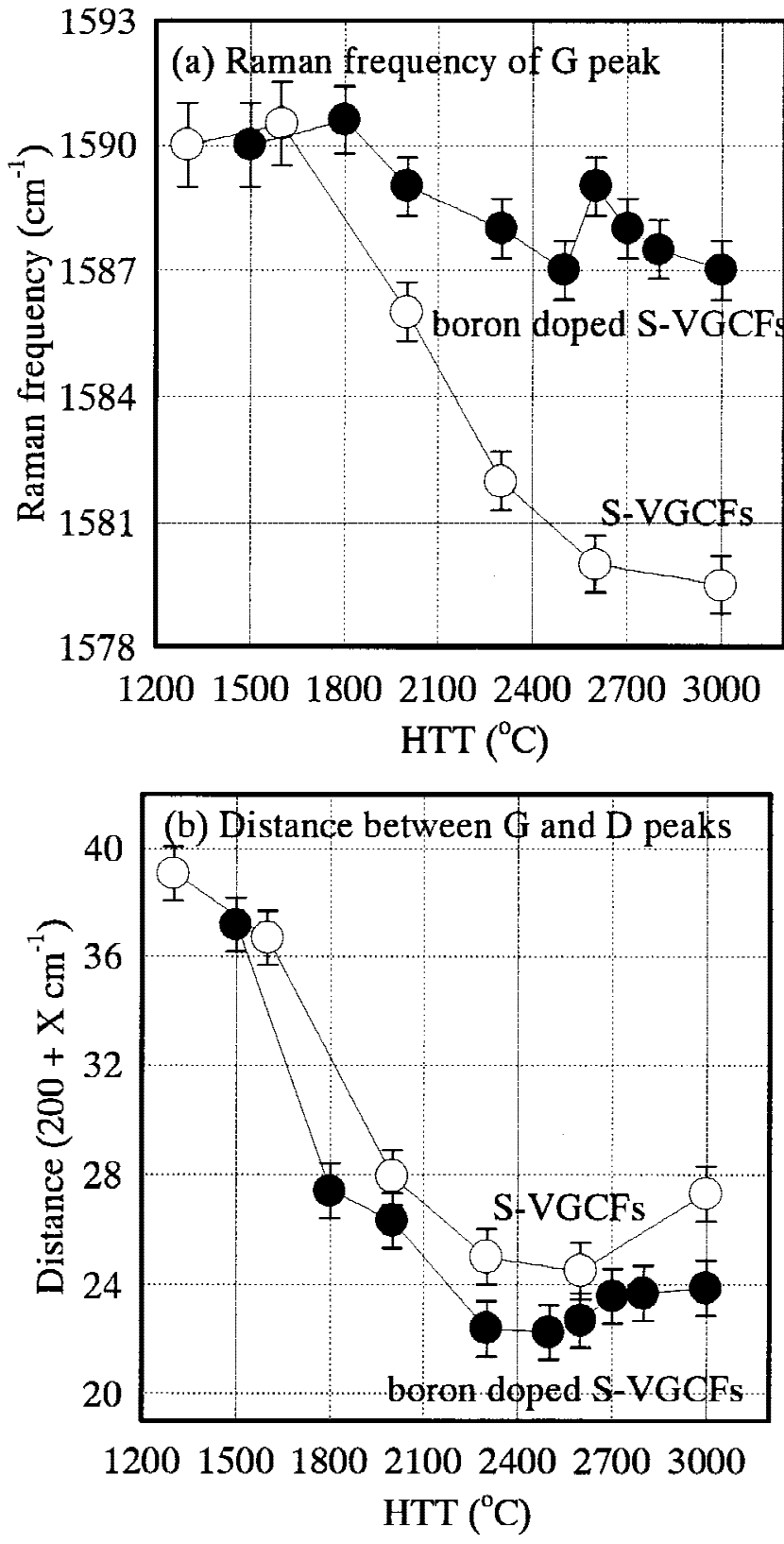

FIG. 7. Variation of (a) the frequency of the G peaks and of (b) the frequency difference between the $G$ and $D$ peaks for undoped and boron-doped S-VGCFs as a function of heat-treatment temperature.

trolyte penetration would be expected. The highly enhanced cyclic efficiency shown in Fig. 13 is related to the reduced degradation of the carbon particles in the anode by the physical binder, and to the abovementioned effects of increased conductivity and electrolyte penetration.

\section{CONCLUSIONS}

S-VGCFs with diameters of $\sim 0.2 \mu \mathrm{m}$ were successfully synthesized by a floating reactant method, using ferrocene as the catalyst. The structural evolution of the
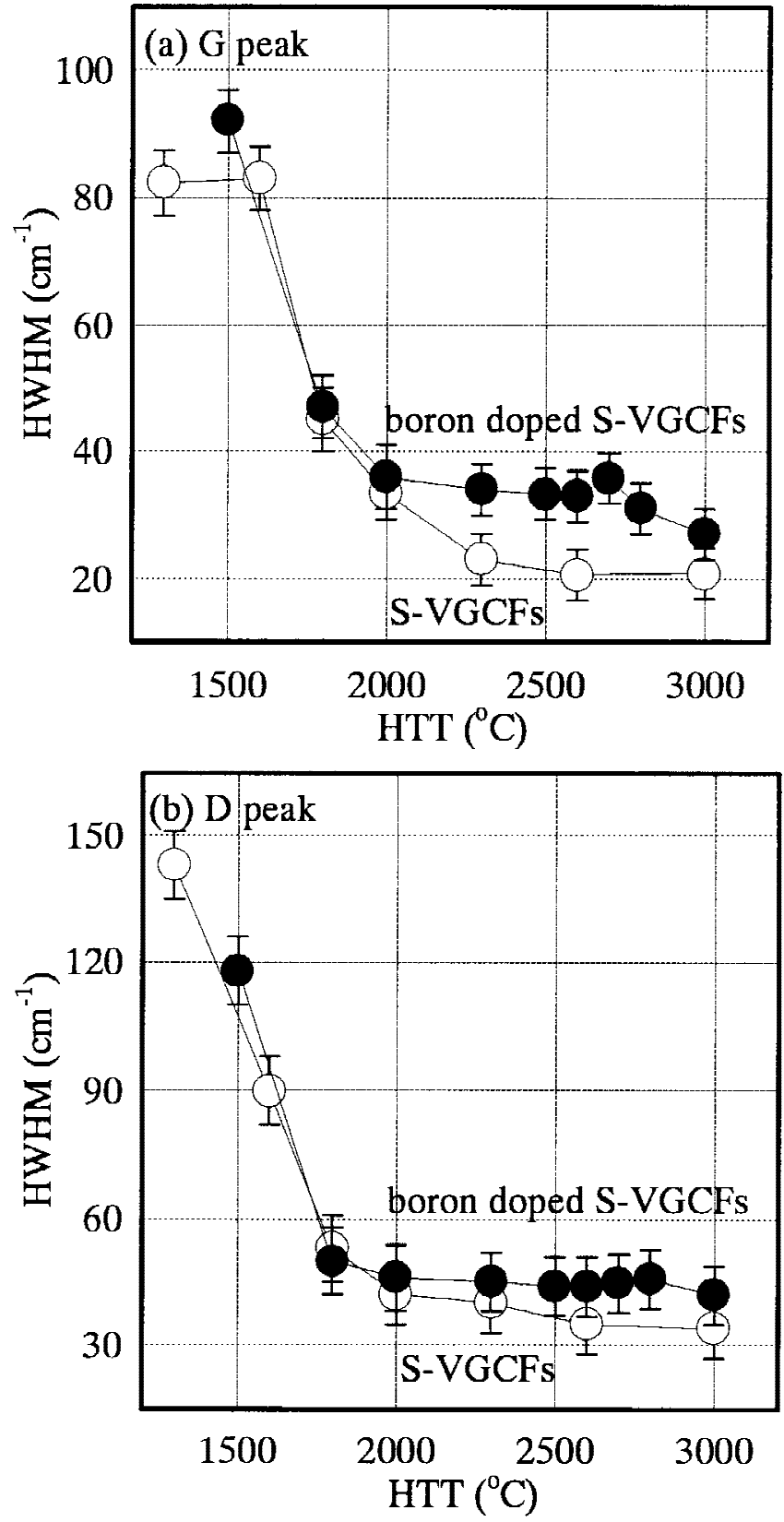

FIG. 8. Variation of the HWHM linewidth of (a) the G peak and (b) the D peak for undoped and boron-doped S-VGCFs as a function of heat-treatment temperature.

undoped and boron-doped S-VGCFs have been characterized as a function of HTT using XRD, scanning electron microscopy (SEM), and microprobe Raman spectroscopy. The anode performance of both types of sample was measured, and also the effects of S-VGCF addition to synthetic graphite for anode performance was evaluated.

Based on the XRD measurements, we conclude that boron atoms act as a catalyst for enhancing the graphitization process for the HTT range from 1800 to $2500^{\circ} \mathrm{C}$, possibly due to the higher diffusivity of boron atoms over 


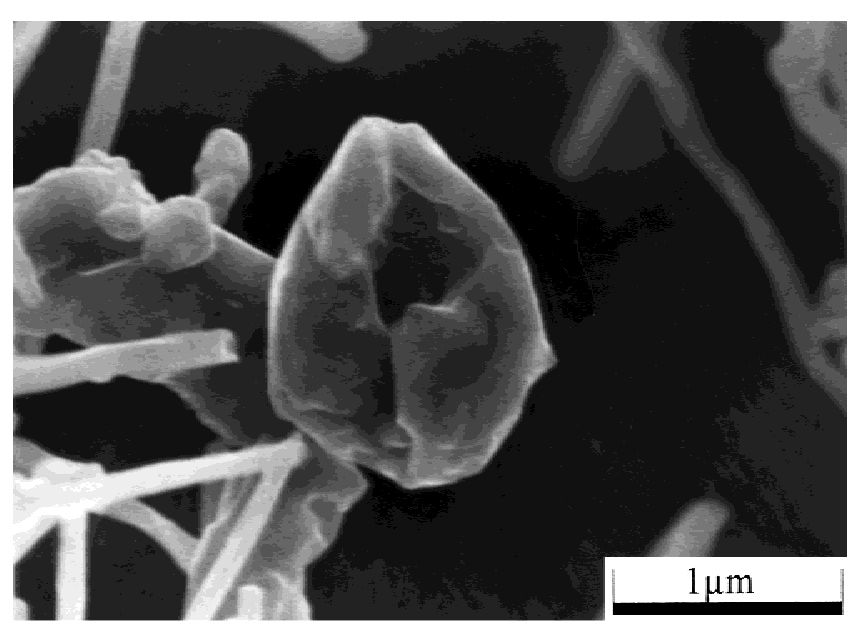

(a)

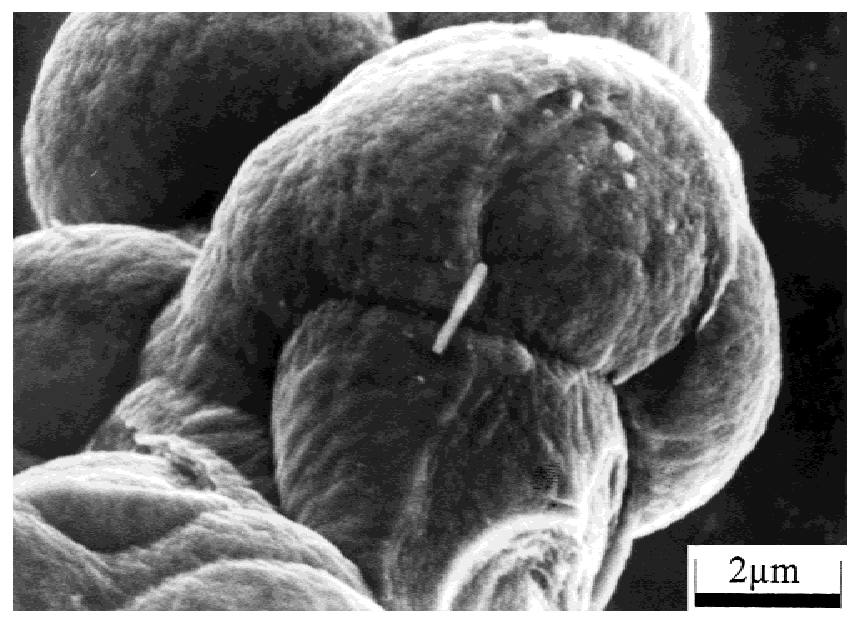

(b)

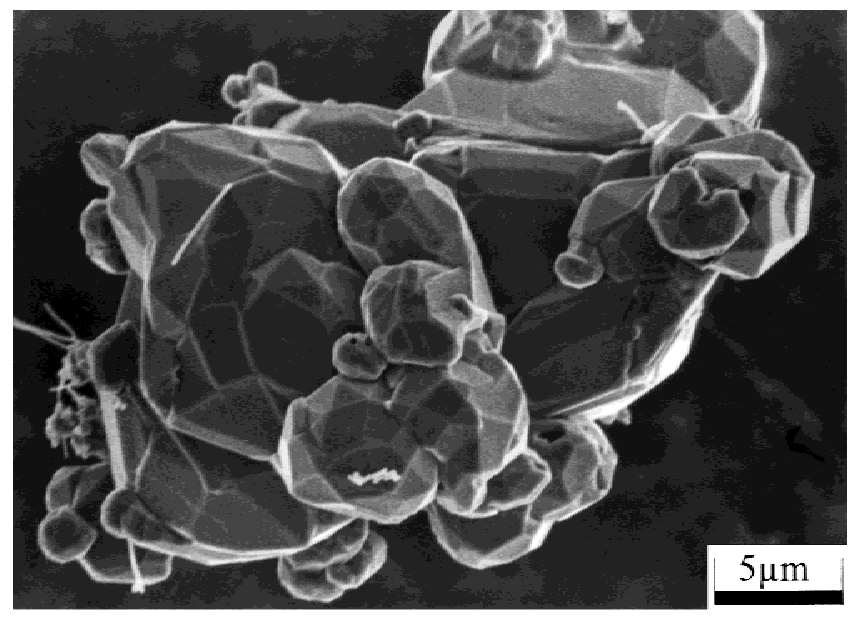

(c)

FIG. 9. FE-SEM photographs of the byproducts formed during the boron-doping process: (a) HTT $=2300{ }^{\circ} \mathrm{C}$, (b) $\mathrm{HTT}=2600{ }^{\circ} \mathrm{C}$, and (c) $\mathrm{HTT}=3000^{\circ} \mathrm{C}$.

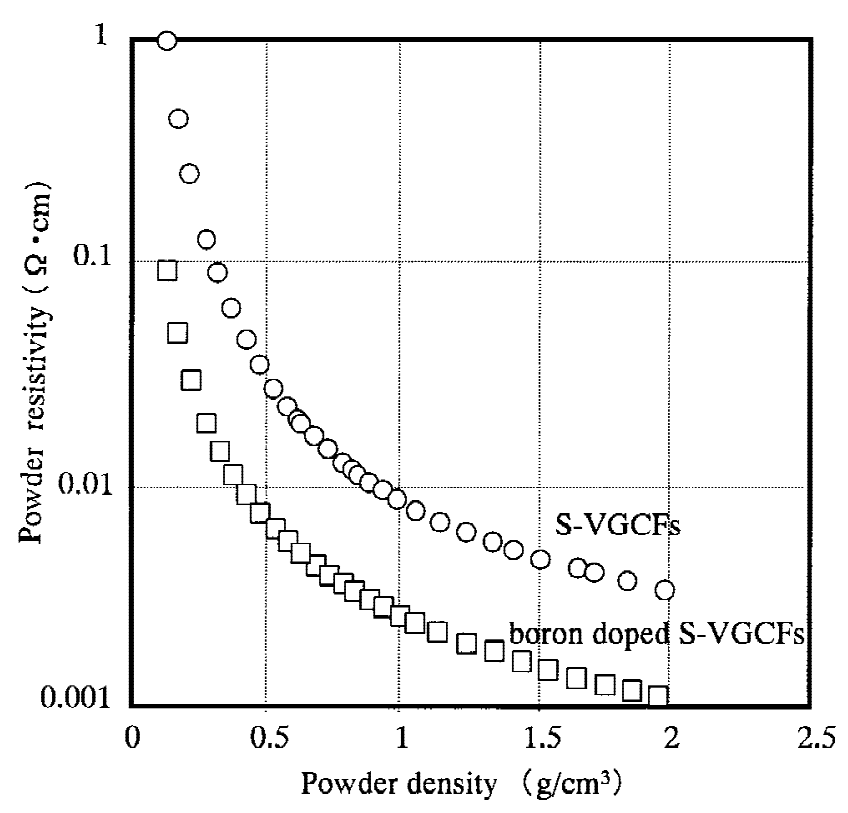

FIG. 10. Powder resistivity of undoped and boron-doped S-VGCFs as a function of powder density.

carbon atoms. The physical mechanism responsible for this behavior is the larger $\mathrm{B}-\mathrm{C}$ bond length compared to that for the $\mathrm{C}-\mathrm{C}$ bond, which causes increased strain at higher HTT, therefore causing a release of B from substitutional sites, increased boron out-diffusion from the host material, and an increase in the density of vacancy defects in the lattice. The depolarized ratio $\left(D_{\mathrm{P}}=I_{\mathrm{V}} / I_{\mathrm{P}}\right)$ of the $\mathrm{G}$ peak in the Raman spectra gives very important information regarding variation of the crystallite size caused by boron doping. The boron-doped S-VGCFs show the same behavior of $D_{\mathrm{p}}$ as compared with that of undoped S-VGCFs for the HTT range below $2300{ }^{\circ} \mathrm{C}$, but deviations start to occur at $\mathrm{HTT} \approx 2500{ }^{\circ} \mathrm{C}$, approaching a minimum value in $D_{\mathrm{p}}$ for the sample with HTT $=2700{ }^{\circ} \mathrm{C}$, and, finally, an increase in $D_{\mathrm{p}}$ with increasing HTT is observed. In this study, we focused on the disorder caused by boron atoms at higher HTT. As a result, the decrease in the depolarized ratio is identified with the boron out-diffusion process, which in turn gives rise to an increased density of defects, such as tilt boundary defects. Furthermore, the results for the depolarized ratios are closely consistent with the XRD results even though the two analytical techniques have different detection depths, XRD having a large depth and Raman scattering a much shorter depth. This kind of consistency might be related to particular features of S-VGCFs, such as their small diameter. That is, boron atoms can easily diffuse into and out of S-VGCFs.

In terms of anode capacity in lithium ion batteries, boron-doped S-VGCFs show an improved capacity of $351 \mathrm{mAh} / \mathrm{g}$ and higher Coulombic efficiency of $81.1 \%$ as compared with the corresponding values for undoped 

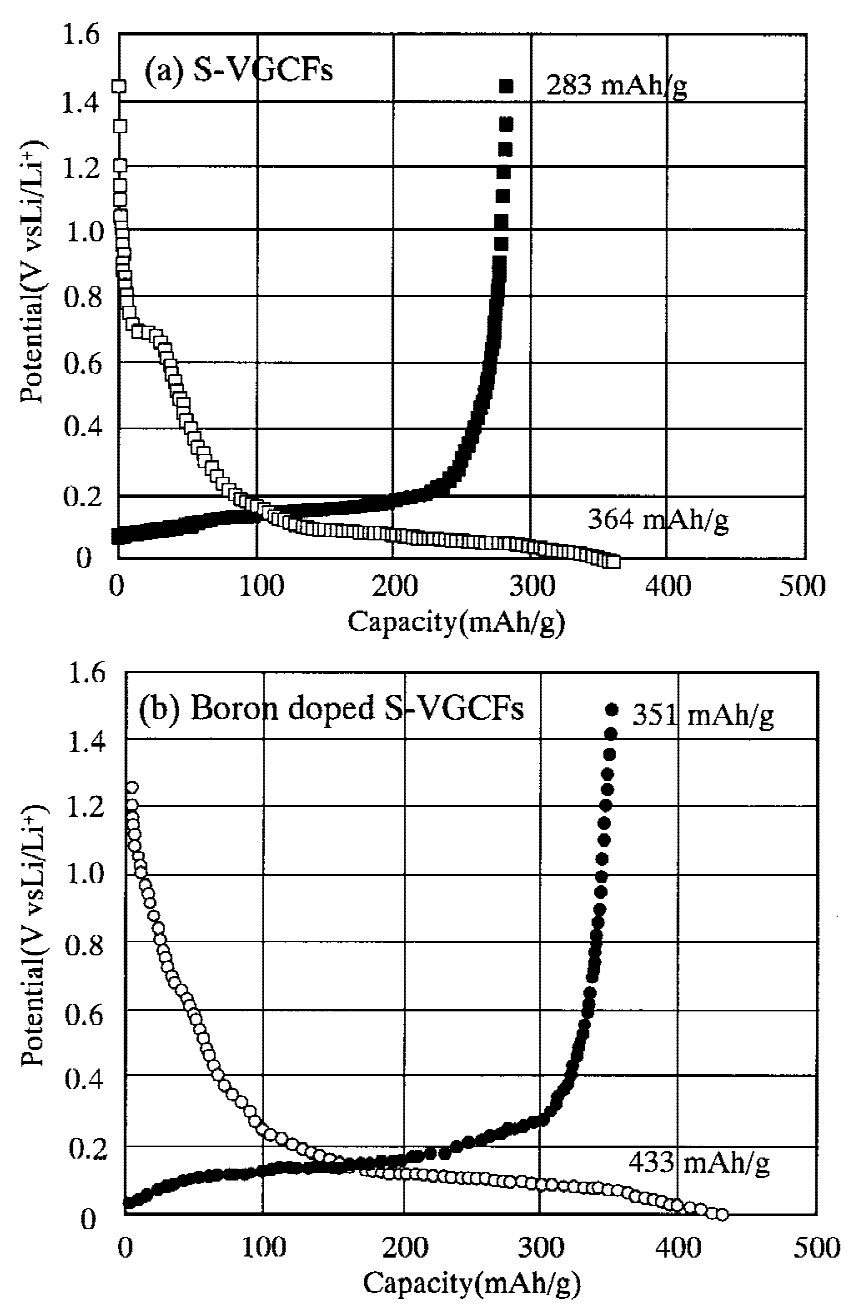

FIG. 11. Charge and discharge curves for (a) undoped S-VGCFs and (b) boron-doped S-VGCFs during the first cycle: lattice constant $C_{\mathrm{o}}$ $6.777 \AA$ Å $6.753 \AA$, respectively; Coulombic efficiency, $77.7 \%, 81.8 \%$, respectively.

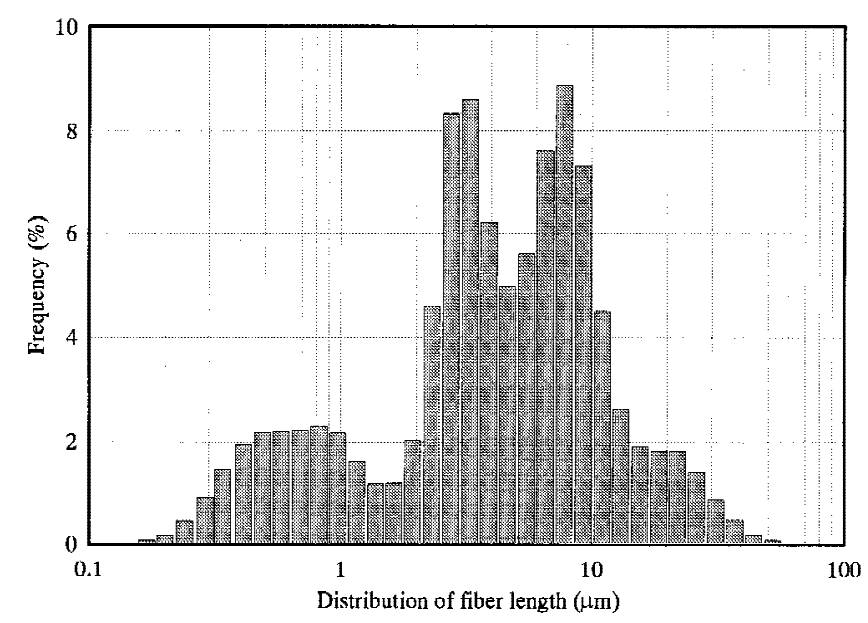

FIG. 12. Length distribution of S-VGCFs $\left(\mathrm{HTT}=3000^{\circ} \mathrm{C}\right)$ measured by a laser particle size analyzer.

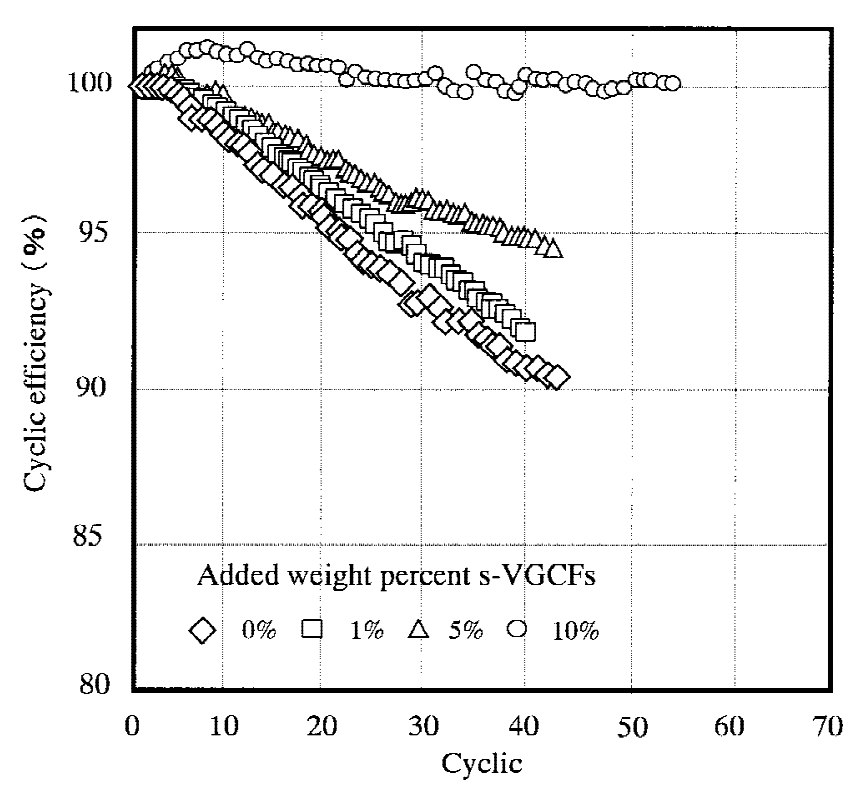

FIG. 13. Cyclic efficiency of synthetic graphite $\left(\mathrm{HTT}=2900^{\circ} \mathrm{C}\right)$ as a function of added weight percent of S-VGCFs; cycle characteristic of $0.2 \mathrm{~mA} / \mathrm{cm}^{2}$ at $0-1.5 \mathrm{~V}$.

S-VGCFs (283 mAh/g, 77.7\%). These results cannot be explained only by the presence of boron atoms in substitutional sites acting as a catalyst for graphitization, but rather, the defects caused by the boron out-diffusion process, such as tilt boundary defects, could contribute to the increase in the capacity and decrease in the powder resistivity.

When S-VGCFs were used as an additive to synthetic graphite, the cyclic efficiencies were improved with increasing weight percent of S-VGCFs, and, in particular, for the case of $10 \mathrm{wt} \% \mathrm{~S}$-VGCF addition, the composite showed a cyclic efficiency of almost $100 \%$. This result indicates that S-VGCFs act as a physical binder for synthetic graphite to inhibit the degradation of the anode material through charge-discharge cycles, while increasing the bulk conductivity and the electrolyte penetration.

\section{ACKNOWLEDGMENT}

Part of the work by M. Endo was supported by a grant-in-aid for "Research for the Future Program," Nano-carbon, from the Japan Society for the Promotion of Science.

\section{REFERENCES}

1. T. Koyama, Carbon 10, 757 (1972).

2. M. Endo and M. Komaki, in Ext. Abst. 16th Biennial Conf. (American Carbon Society, San Diego, CA, 1983), p. 523.

3. A. Oberlin, M. Endo, and T. Koyama, J. Cryst. Growth 32, 335 (1972).

4. J.S. Speck, M. Endo, and M.S. Dresselhaus, J. Cryst. Growth 94, 834 (1989).

5. G.G. Tibbetts, Appl. Phys. Lett. 42, 666 (1983). 
6. F. Benissad, P. Gadelle, M. Coulon, and L. Bonnetain, Carbon 26, 61 (1988).

7. G.G. Tibbetts, D.W. Gorkiewicz, and R.L. Alig, Carbon 30, 809 (1993).

8. M. Ishioka, T. Okada, K. Matsubara, and M. Endo, Carbon 11, 674 (1992).

9. B.M. Way, J.R. Dahn, T. Teidje, K. Myrtle, and M. Kasrai, Phys. Rev. B 46, 1697 (1992).

10. J. Kouvetakis, R.B. Kaner, M.L. Sattler, and N. Bartlett, J. Chem. Soc., Chem. Commun. 1758 (1986).

11. M. Chesneau, F. Beguin, J. Conard, R. Erre, and J. Thebault, Carbon 30, 714 (1992).

12. D.L. Fecko, L.E. Jones, and P.A. Thrower, Carbon 31, 637 (1993).

13. D. Tomanek, R.M. Wentzcovitch, S.G. Louie, and M.L. Cohen, Phys. Rev. B 37, 3134 (1988).

14. Q. Wang, X. Ma, L-Q. Chen, W. Cermignani, and C.G. Pantano, Carbon 35, 307 (1997).
15. C.E. Lowell, J. Am. Ceram. Soc. 50, 142 (1967).

16. T. Hagio, M. Nakamizo, and K. Kobayashi, Carbon 25, 637 (1987).

17. M. Endo, C. Kim, T. Karaki, Y. Nishimura, M.J. Matthews, S.D.M. Brown, and M.S. Dresselhaus, Carbon 37, 561 (1999).

18. M. Endo, CHEMTECH 8, 568 (1988).

19. Joint Committee for Powder Diffraction, Standard File No. 40, 1995.

20. M. Yoshikawa, N. Nagai, M. Matsuki, H. Fukuda, G. Katagiri, H. Ishida, and A. Ishitani, Phys. Rev. B 1992, 46, 7169 (1992).

21. H.W. Schrotter, in Raman Spectroscopy (Plenum, New York, 1970), p. 69.

22. G.R. Hennig, J. Chem. Phys. 42, 2267 (1965).

23. T.C. Chieu, M.S. Dresselhaus, and M. Endo, Phys. Rev. B. 26, 5867 (1982).

24. P. Lespade, A. Marchand, M. Couzi, and F. Cruege, Carbon 22, 375 (1984).

25. F. Tuinstra and J.L. Koenig, J. Chem. Phys. 53, 1126 (1970). 